\title{
长效、经济的发展问题保真 计算格式反演补偿*
}

\author{
钟 青 \\ (中国科学院大气物理研究所, 北京 100029)
}

\section{关链词 反滨补偿构造原理、保真格式、气候漂移、长效、经济}

继瞬时平方守恒格式出现 ${ }^{[1]}$ 以来, 现在已能构造完全的隐式 ${ }^{[2,3]}$ 、显式 ${ }^{[2-4]}$ 和半隐式 ${ }^{[2]}$ 平方 守恒格式. 本文在吸取了加 “可调”耗散项构造显式完全平方守桓格式 ${ }^{[4]}$ 思想合理内核，以及 采用 ‘瞬时线性化”方法实现隐式完全平方守恒格式 ${ }^{[3]}$ 思想而建立的半隐式(含隐式与显式)完 全平方守恒格式理论 ${ }^{[2,5-7]}$ 的框架基础上,进一步提出了按照离散计算误差引入的来源与方式, 在离散计算的每一个计算分量(格点)上,以保持原问题单(多)特征性质为原则,对离散计算误 差加以相应平均补偿消除的单 (多) 保真格式叫一般补偿构造原理和反演补偿构造方法, 并具 体实现了若千正压原始方程 (组) 的平方或非平方守恒性质的半隐式或显式的保真拟谱模式; 尤其值得注意的是, 在比较数值实验中发现保真模式具有在经济计算方面, 在延长计算时效与 解决相应“气候漂移”问题方面的作用. 本文工作原理和方法对控制方程无特殊要求, 适用于 平方和非平方守恒性质, 适用于半隐式、显式与隐式格式, 适用于单个与多个特征性质保真, 适 用于任意阶时间差分精度格式.

对于发展问题算子方程

$$
\frac{\partial u}{\partial t}+A u=0
$$

相应补偿保真格式可以写为

$$
\delta u^{n}+\left(A^{n}-A_{L}^{n}\right) u^{n}+A_{L}^{n} t^{n}+\Delta t\left(B_{1}^{n}+\varepsilon^{*} B_{2}^{n}\right) u^{n}-0 .
$$

这里, 若辅助构造算子 $A_{2}$ 分别取为算子 $A$ 的线性(化)部分, 零或 $A$ 本身则格式(2)分别为半 隐式,显式或(瞬时线性化)隐式格式; $\varepsilon^{*}$ 为可具有多个分量的待定补偿系数,补偿算子 $B_{1}^{n} u^{n}$, $B_{2}^{*} u^{*}$ 分别可写为

$$
\begin{aligned}
& F_{i}^{*} u^{*}=\left\{\begin{array}{l}
-\sum_{j=1}^{J} \Delta t^{j-1}\left[\frac{1}{(j+1) !}\left(\frac{\partial^{i+1} u}{\partial t^{j+1}}\right)+A_{L}^{n} \frac{1}{j !}\left(\frac{\partial^{j} u}{\partial t^{i}}\right)^{n}\right], J \text { 正整数, } \\
0, J=0,
\end{array}\right. \\
& B_{2}^{n} u^{n}=-\frac{\Delta t^{\prime}}{(J+2) !}\left(-\frac{\partial^{J+2} u}{\partial t^{J+2}}\right)^{n}, J \text { 非负整数, }
\end{aligned}
$$

其中 $\delta u^{*}-\Delta t^{-1}\left(u^{n+1}-u^{n}\right), u$ 的各阶时间导数可由方程 (1) 确定.

“国家!“七五”、“八五”攻关及中国科学院大气物理研究所所长择优基金部分资助项目. 
特别地,具有一般整体平方守恒性质

$$
\int \tilde{A} u \cdot \tilde{A} A u \mathrm{~d} \sigma=0 .
$$

或者具有…般整体加权平方守恒性质

$$
\int\left[2 \tilde{A} u \tilde{A} u \cdot \tilde{A} A u+\tilde{A} A u(\tilde{A} u)^{2}\right] \mathrm{d} \sigma=0
$$

的算子方程(1)成立如下定理, 这里, $\tilde{A}$ 和 $\tilde{A}$ 分别为独立于 $u, t$ 的有界和有界正定空间算 子, $\mathrm{d} \sigma$ 为空间积分元.

定理 1 若误差补偿系数 $\varepsilon^{*}$ 取为

$$
\varepsilon^{n}=\frac{c_{1}}{1+\sqrt{1-\Delta t^{2} c_{1} c_{2}}},
$$

则 (2)式为与(1)式相容的整体平方守恒性质保真格式, 其中 $c_{1}$ 和 $c_{2}$ 分别为

$c \int\left[2 \tilde{A} u^{n} \cdot \tilde{A} M_{4} u^{n}+\left(\tilde{A} M_{1} M_{2} u^{n}\right)^{2}\right] \mathrm{d} \sigma$ 和 $c \int\left(\tilde{A} M_{1} B_{2}^{n} u^{*}\right)^{2} \mathrm{~d} \sigma, c$ 为 $\int \tilde{A} M_{1} B_{2}^{n} u^{n} \cdot \tilde{A} M_{3} u^{n} \mathrm{~d} \sigma$ 的 倒数, $M_{1}$ 为 $I+\Delta t A_{L}^{n}$ 逆算子, $M_{2}, M_{3}$ 和 $M_{4}$ 分别为算子 $A^{n}+\Delta t B_{1}^{n}, I-\Delta t M_{1} M_{2}$ 和 $M_{1} A_{L}^{n} M_{2}-B_{1}^{n}, I$ 为单位算子.

证 利用性质(5),易验证: 若 $\varepsilon^{*}$ 满足(7)式,则任意 $n$ 时成立

$$
\int\left(\tilde{A} u^{n+1}\right)^{2} \mathrm{~d} \sigma-\int\left(\tilde{A} u^{*}\right)^{2} \mathrm{~d} \sigma,
$$

即此时(2)式为整体平方守恒性质保真格式. 又从(7)式显然有

$$
\lim _{\Delta t \rightarrow 0} \varepsilon^{n}=O\left(\Delta t^{0}\right)
$$

即此时(2)式亦与(1)式相容. 证毕.

显然, 整体平方守恒性质保真格式为稳定计算格式. 而其计算稳定的充分必要条件为

$$
1 \geqslant \Delta t^{2} c_{1} c_{2} \text {. }
$$

定理 2 若误差补偿系数 $\varepsilon^{n}$ 满足关系式

$$
\Delta t^{4} \gamma_{1}\left(\varepsilon^{n}\right)^{3}+\Delta t^{2} \gamma_{2}\left(\varepsilon^{n}\right)^{2}+\gamma_{3} \varepsilon^{n}+\gamma_{4}=0,
$$

且量级为 $O\left(\Delta t^{0}\right)$, 则(2)为与(1)相容的整体加权平方守恒性质保真格式. 其中, $\gamma_{1}, \gamma_{2}, \gamma_{3}$ 和 $\gamma_{4}$ 分别为 $\int \tilde{A} M_{1} B_{2}^{n} u^{*}\left(\tilde{A} M_{1} B_{2}^{n} u^{*}\right)^{2} \mathrm{~d} \sigma$,

$-\int\left[2 \tilde{A} M_{1} B_{2}^{n} u^{n} \tilde{A} M_{1} B_{2}^{n} u^{n} \cdot \tilde{A} M_{3} u^{n}+\tilde{A} M_{3} u^{n}\left(\tilde{A} M_{1} B_{2}^{n} u^{n}\right)^{2}\right] \mathrm{d} \sigma$,

$\int\left[2 \tilde{A} M_{3} u^{n} \tilde{A} M_{1} B_{2}^{n} u^{n} \cdot \tilde{A} M_{3} u^{n}+\tilde{A} M_{1} B_{2}^{n} u^{n}\left(\tilde{A} M_{3} B_{2}^{n} u^{n}\right)^{2}\right] \mathrm{d} \sigma$ 和

$-\int\left[2 \tilde{A} M_{1} M_{2} u^{n} \tilde{A} M_{1} M_{2} u^{n} \cdot \tilde{A} u^{n}+2 \tilde{A} u^{n} \tilde{A} M_{4} u^{n} \cdot \tilde{A} u^{n}+\tilde{A} M_{3} u^{n}\left(\tilde{A} M_{1} M_{2} u^{n}\right)^{2}+\right.$ $\left.\tilde{A} M_{4} u^{n}\left(\tilde{A} u^{n}\right)^{2}\right] \mathrm{d} \sigma$; 且 $1, M_{1}-M_{4}$ 定义同定理 1 .

证 利用性质(6), 易验证: 若 $\varepsilon^{*}$ 满足(10)式,则任意 $n$ 时刻成立

$$
\int \tilde{A} u^{*+1}\left(\tilde{A} u^{*+1}\right)^{2} d \sigma-\int \tilde{A} u^{n}\left(\tilde{A} u^{n}\right)^{2} \mathrm{~d} v,
$$

即此时(2)式为整体加权平方守恒性质保真格式. 又从(11)式显然有 


$$
\lim _{\Delta t \rightarrow 0} \varepsilon_{*}-\lim _{\Delta t \rightarrow 0}\left(\frac{\gamma_{1}}{\gamma_{3}}-\Delta t^{2} \frac{\gamma_{2}}{\gamma_{3}}-\Delta t^{4} \frac{\gamma_{1}}{\gamma_{3}}\right)=\lim _{\Delta t \rightarrow 0} \frac{\gamma_{3}}{\gamma_{1}}-O\left(\Delta t^{0}\right),
$$

即此时(2)式亦与(1)式相容. 证毕.

显而易见, 整体加权平方守恒性质保真格式为绝对稳定的计算格式.

此外,关于格式(2)的计算收敛性证明,限于篇幅将另文发表.

考虑地球球面坐标系里的正压原始方程组

$$
\begin{aligned}
& \left\{\begin{array}{l}
\frac{\partial u}{\partial t}--\frac{1}{a \cos \varphi}\left(\frac{\partial \Phi^{\prime}}{\partial \lambda}+u \frac{\partial u}{\partial \lambda}+V \frac{\partial u}{\partial \varphi}\right)+\left(f+\frac{u \operatorname{tg} \varphi}{a}\right) \nu, \\
\frac{\partial v}{\partial t}-\frac{1}{a \cos \varphi}\left(\frac{\partial \Phi^{\prime}}{\partial \varphi}+u \frac{\partial \nu}{\partial \lambda}+V \frac{\partial \nu}{\partial \varphi}\right)-\left(f+\frac{u \operatorname{tg} \varphi}{a}\right)^{u}, \\
\frac{\partial \Phi^{\prime}}{\partial t}=-\frac{1}{a \cos \varphi}\left[\frac{\partial}{\partial \lambda}\left(u \Phi^{\prime}\right)+\frac{\partial}{\partial \varphi}\left(V \Phi^{\prime}\right)\right]-\Phi D,
\end{array}\right. \\
& \frac{\partial \zeta}{\partial t}=F \zeta, \\
& \text { 或 } \frac{\partial D}{\partial t}-F D-\nabla^{2} \Phi^{\prime} \text {, } \\
& \frac{\partial \Phi^{\prime}}{\partial t}-F \Phi-\Phi D,
\end{aligned}
$$

其中非线性项 $F \zeta, F D$ 和 $F \Phi$ 分别为 $\frac{1}{a \cos ^{2} \varphi}\left(\frac{\partial F \nu}{\partial \lambda}-\cos \varphi \frac{\partial F u}{\partial \varphi}\right)$,

$$
\begin{gathered}
-\frac{1}{a \cos ^{2} \varphi}\left(\frac{\partial F u}{\partial \lambda}+\cos \varphi \frac{\partial F v}{\partial \varphi}\right), \\
-\nabla^{2} \frac{u^{2}+\nu^{2}}{2} \text { 和 }-\frac{1}{a \cos ^{2} \varphi}\left[\frac{\partial\left(U \Phi^{\prime}\right)}{\partial \lambda}+\cos \varphi \frac{\partial\left(V \Phi^{\prime}\right)}{\partial \varphi}\right] ; F u-U(\zeta+f), F v-V(\zeta+f) ; \\
U-u \cos \varphi, V=v \cos \varphi .
\end{gathered}
$$

方程(13)相应补偿保真与气象传统(拟)谱半隐式模式可以分别写为

$$
\begin{aligned}
& \left\{\begin{array}{l}
\delta \zeta_{i m}^{n}-F \zeta_{i m}^{*}+\varepsilon^{*} B \zeta_{m l}^{n}, \\
\delta D_{l m}^{n}-F D_{i m}^{n}+\frac{l(l+1)}{a^{2}} \Phi_{i m}^{n+1}+\varepsilon^{*} B D_{l_{m}}^{n}, \\
\delta \Phi_{i_{m}^{\prime}}^{n}-F \Phi_{i m}^{n}-\Phi D_{i m}^{n+1}+\varepsilon^{*} B \Phi_{i m}^{n},
\end{array}\right.
\end{aligned}
$$

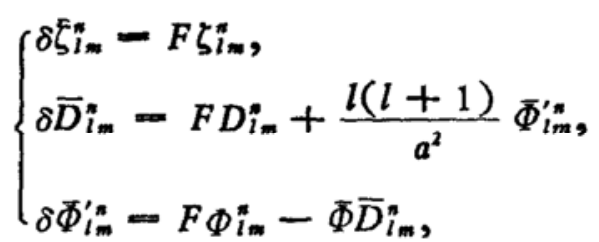

这里, $\delta \bar{X}^{*}, \bar{X}^{*}$ 分别为 $0.5 \Delta t^{-1}\left(X_{l m}^{n+1}-X_{l m}^{n-1}\right), 0.5\left(X_{l m}^{n+1}+X_{i m}^{-1}\right) ; X_{l m}^{n}$ 表示为变量 $X$ 在 $n$ 时刻以球谐系数 $P_{l}^{m}(\mu) e^{i m \lambda}$ 为基函数相应拟谱截断展开谱系数; 且作为补偿原理的初步应 用, $B X$ 可作为 $X^{\prime \prime}-2 X^{\prime}+X^{*}, X^{\prime}$ 和 $X^{\prime \prime}$ 可通过取 (14)式中 $\varepsilon^{*}=0$, 然后以 $X^{*}$ 为初 值将 (14)式分别以 $\Delta t$ 为步长向前积分一步和二步获得, 其中 $X-\left\{\zeta, D, \Phi^{\prime}\right\}$. 进一步分别 以保持 (12)或 (13)式中相应整体性质为原则, 确定 $\varepsilon^{*}$. 本工作分别实现了能量、拟能和角动 


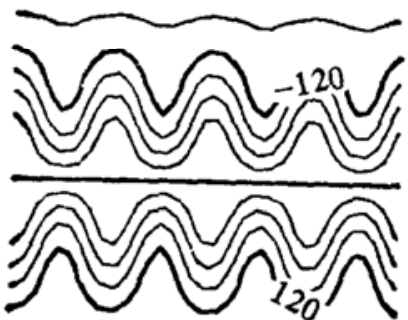

图1 初值 $\mathrm{R}-\mathrm{H}$ 波

$\operatorname{Min}=-0.150000 E+9$,

Max $=0.15000 \mathrm{E}+9$,

INT. $=0.30000 \mathrm{E}+8$

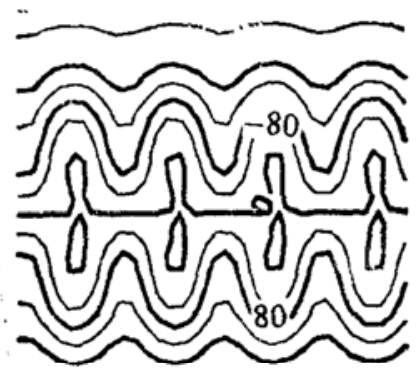

图 2 计算的第 68 天 $\mathrm{R}-\mathrm{H}$ 波 (传 统拟谱模式,积分步长 $10 \mathrm{~min}$ )

$\mathrm{Min}=-0.20000 \mathrm{E}+9$,
$\mathrm{Max}=0.20000 \mathrm{E}+9$,
$\mathrm{INT} .=0.40000 \mathrm{E}+8$

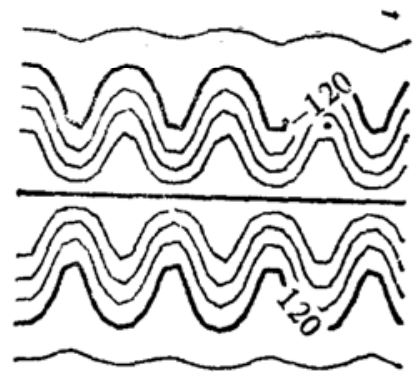

图 3 计算的第90天 $\mathrm{R}-\mathrm{H}$ 没(动 能保真拟谱模式, 积分步长 $3 \mathrm{~h}$ )
$\mathrm{Min}=-0.15000 \mathrm{E}+9$,
$\mathrm{Max}=0.15000 \mathrm{E}+9$,
INT. $=0.30000 \mathrm{E}+8$

表 1 动能和拟能全球积分守恒量计算变化・"

\begin{tabular}{c|l|l}
\hline 天 & 动能守恒量 & 拟能守恒摄 \\
\hline 0 & $0.1220760 \mathrm{E}+5$ & $0.8847784 \mathrm{E}-8$ \\
30 & $0.1220771 \mathrm{E}+5$ & $0.8847945 \mathrm{E}-8$ \\
35 & $0.1220796 \mathrm{E}+5$ & $0.8848321 \mathrm{E}-8$ \\
40 & $0.1220877 \mathrm{E}+5$ & $0.8849525 \mathrm{E}-8$ \\
45 & $0.1221150 \mathrm{E}+5$ & $0.8853549 \mathrm{E}-8$ \\
50 & $0.1222102 \mathrm{E}+5$ & $0.8867624 \mathrm{E}-8$ \\
65 & $0.1225634 \mathrm{E}+5$ & $0.8919797 \mathrm{E}-8$ \\
65 & $0.1241432 \mathrm{E}+5$ & $0.9152241 \mathrm{E}-8$ \\
70 & $0.1348819 \mathrm{E}+5$ & $0.1071767 \mathrm{E}-8$ \\
\hline
\end{tabular}

a) 积分步长 $10 \mathrm{~min}$, 不含重力波过程的正压原始方程传统拟谱模式, 单精度.

表 2 能量、拟能与角动量全球积分守恒量计算变化")

\begin{tabular}{|c|c|c|c|c|c|}
\hline 天 & 能量守恒量 & 天 & 找能守恒量 & 湥 & 角动量守恒是 \\
\hline 0 & $0.22205265 E+10$ & 0 & $0.3151571 \mathrm{E}-11$ & 0 & $0.9140436 \mathrm{E}+9$ \\
\hline 200 & $0.22205265 E+10$ & 50 & $0.3151572 \mathrm{E}-11$ & 50 & $0.9140437 \mathrm{E}+9$ \\
\hline 400 & $0.22205270 E+10$ & 100 & $0.3151572 \mathrm{E}-11$ & 100 & $0.9140439 E+9$ \\
\hline 600 & $0.22205265 E+10$ & 250 & $0.3151571 \mathrm{E}-11$ & 120 & $0.9140437 \mathrm{E}+9$ \\
\hline 700 & $0.22205265 \mathrm{E}+10$ & 400 & $0.3151571 \mathrm{E}-11$ & 140 & $0.9140435 E+9$ \\
\hline 750 & $0.22205267 E+10$ & 420 & $0.3151572 E-11$ & 160 & $0.9140437 E+9$ \\
\hline 780 & $0.22205265 \mathrm{E}+10$ & 450 & $0.3151571 \mathrm{E}-11$ & 180 & $0.9140437 E+9$ \\
\hline 800 & $0.22205265 \mathrm{E}+10$ & 800 & $0.3151571 \mathrm{E}-11$ & 190 & $0.9140434 E+9$ \\
\hline
\end{tabular}

a) 积分步长 6,5 与 $3 \mathrm{~h}$, 含重力波过程的正压原始方程单保真模式, 单精度,

量整体守恒性质完全保真格式(14).

数值积分结果表明: 传统格式(15)中存在系统误差, 其能量、拟能和角动量全球积分相对 误差均呈振荡变化-对称振荡增长-单调正增长(计算失隐)规律; 特别地, 无辐散 $(D=0)$ 情 形下, 关于准确解 4 波数 Rossby-Haurwitz 波(见图 1), 传统格式(15)计算波型基本保持时 间约为 60 天左右 $(<70$ 天)(参见表 1 、图 2), 并且不能通过减小时间步长改变误差演变规律 
表 3 动能和拟能全球积分守恒量计算变化 ")

\begin{tabular}{r|l|l}
\hline 天 & 动能守佰量 & 㮸能守恒量 \\
\hline 30 & $0.12207602 \mathrm{E}+5$ & $0.8847784 \mathrm{E}-8$ \\
35 & $0.12207600 \mathrm{E}+5$ & $0.8847788 \mathrm{E}-8$ \\
40 & $0.12207602 \mathrm{E}+5$ & $0.8847789 \mathrm{E}-8$ \\
45 & $0.12207602 \mathrm{E}+5$ & $0.8847784 \mathrm{E}-8$ \\
50 & $0.12207601 \mathrm{E}+5$ & $0.8847786 \mathrm{E}-8$ \\
55 & $0.12207601 \mathrm{E}+5$ & $0.8847789 \mathrm{E}-8$ \\
60 & $0.12207602 \mathrm{E}+5$ & $0.8847784 \mathrm{E}-8$ \\
65 & $0.12207604 \mathrm{E}+5$ & $0.8847788 \mathrm{E}-8$ \\
70 & $0.12207602 \mathrm{E}+5$ & $0.8847786 \mathrm{E}-8$ \\
75 & $0.12207604 \mathrm{E}+5$ & $0.8847784 \mathrm{E}-8$ \\
80 & $0.12207600 \mathrm{E}+5$ & $0.8847784 \mathrm{E}-8$ \\
85 & $0.12207601 \mathrm{E}+5$ & $0.8847789 \mathrm{E}-8$ \\
90 & $0.12207601 \mathrm{E}+5$ & $0.8847786 \mathrm{E}-8$ \\
95 & $0.12207604 \mathrm{E}+5$ & $0.8847788 \mathrm{E}-8$ \\
100 & $0.12207601 \mathrm{E}+5$ & $0.8847791 \mathrm{E}-8$ \\
\hline
\end{tabular}

2) 积分步长 $3 \mathrm{~h}$, 不含重力波过程的正压原始方程动能保真模式,单精度.

以及延长此计算“时效”. 而保真格式(14)则在计算机舍人误差水平上确可保真(参见表 2); 特 别地, 无辐散 $(D-0)$ 情形下, 关于准确解 4 波数 Rossby-Haurwitz 波, 无论拟能还是能量 保真格式 (14)都可在计算机舍人误差水平上解决非线性计算稳定性和收敛性问题(参见表 $3 、$ 图 3). 比较传统与保真格式表 3、图 3 与表 1、图 2 数值实验结果还显示: 保真格式还具有经 济计算, 延长相应传统格式计算时效, 解决其系统谣差造成的计算失效问题或 “气候漂移”问题 方面的作用. 本文及文献 $[2,4-7]$, 还显示时间差分离散误差很小不重要的传统观念 ${ }^{[8]}$ 需重 新考虑.

本文计算中,地球球面上选取经向 32 个等距格点, 纬向 26 个不等距高斯格点. 球谐函数 展开采用菱形截谱方法,最大截断波数 11. 文中数值实验均是在中国科学院大气物理研究所 Convex-1 型数字计算机上进行的,主程序均采用单精度 (7 位有效数字), 子程序采用双精度.

[1] Chang. J., General Circulation Models of the Atmosphere, Academic Press. New York, 1977.

[2] 钟肯, 科学通报, $1991,36(19): 1480 \cdots 1483$.

[3] 曾庆存、张学洪, 中国科学, B 辑, 1981, (11): 1355-1366.

[4]王豩、秀仲贞,科学通报, 1990,35(10):766-768.

[5] 钟青,中国科协首届青年学术年会论文集(理科分册),1992,473-479.

[6] Zhong Qing, CAS/JSC Working Group of Numerical Experiments, Research Activities in Atmosphe. ric and Oceanic Modelling, Report No. 17, 1992, 3, 26.

[7] 钟青,计算物理（失识计算物理学会成立十周年专辑・续二),1992,(4): 758-764.

[8]蒋伯诚、周振中、常说顾,计算物理中的谱方法—FFT 及其应用, 湖南科学技术出版社, 1989. 